Paidéia, 2003, 13(25), 59-72

\title{
DESENVOLVIMENTO DE BEBÊS NASCIDOS PRÉ-TERMO NO PRIMEIRO ANO DE VIDA ${ }^{1}$
}

\author{
Maria Beatriz Martins Linhares ${ }^{2}$ \\ FMRP - Universidade de São Paulo \\ Ana Emília Vita Carvalho \\ FFCLRP - Universidade de São Paulo \\ Carolina Machado \\ CECH. Universidade Federal de São Carlos \\ Francisco Eulógio Martinez \\ FMRP - Universidade de São Paulo
}

\begin{abstract}
Resumo: O presente estudo teve por objetivo avaliar o desenvolvimento, no primeiro ano de vida, de bebês nascidos pré- termo e muito baixo peso. Foram avaliados, através da Escala de Desenvolvimento do Comportamento da Criança (EDCC), 42 bebês nascidos com menos de 34 semanas de idade gestacional e peso igual ou abaixo de 1500g, integrantes do Programa de Seguimento Longitudinal do Desenvolvimento de Bebês Nascidos Prematuros do HCFMRP. Foram identificados eventos psico-sociais adversos no ambiente familiar e representações e expectativas maternas quanto ao desenvolvimento do bebê. $O$ desempenho no EDCC aos 6 e 11 meses de idade classificou-se predominantemente Bom ou Excelente, verificando-se cerca de 35\% de crianças com sinais de Risco ou Atraso. Em que pesem variáveis psicossociais adversas no ambiente familiar, a evolução dos bebês foi positiva no primeiro ano de vida, contextualizada por expectativas e avaliações maternas positivas acerca do bebê e adesão ao programa de follow-up.
\end{abstract}

Palavras-Chave: muito baixo peso ao nascer; prematuridade; desenvolvimento; comportamento; seguimento longitudinal

\section{DEVELOPMENT OF PRE-TERM INFANTS IN THE FIRST-YEAR OF LIFE}

Abstract: The aim of this study was to assess early development of pre-term and low birth weight infants. Forty- two infants born under 34 weeks of gestational age and 1.500 grams were assessed through Developmental and Behavior Child Scale (DBCS) in the first year of life. They have been participated at Developmental Follow-up Program for Pre-term Infants in Clinical Hospital of Faculty of Medicine (USP). Negative psychosocial events in familiar environment, and maternal conceptions and expectations related with child development were evaluated through interview. Performance of children at 6 and 11 months was classified as Good or Excellent, identifying about 35\% of infants with Risk or Delay scores. In spite of psychosocial adversity events in familiar environment, positive child development in the first year of life was observed, related with positive maternal expectations about child development and adherence to a follow-up program.

Key-words: pre-term; very low birth weight; development; behavior; follow-up

\footnotetext{
${ }^{1}$ Artigo recebido para publicação em 09/03/03; aceito em 06/05/03.

${ }^{2}$ Endereço para correspondência: Maria Beatriz Martins Linhares, departamento de Neurologia, Psiquiatria e Psicologia Médica, Faculdade de Medicina de Ribeirão Preto, USP, Av. Nove de Julho, 980, Ribeirão Preto, SP, Cep 14025-000, E-mail: Linhares@fmrp.usp.br
} 
Estudos sobre fatores de risco na infância incluem a prematuridade, o muito baixo peso ao nascer (abaixo de 1.500 gramas) e as complicações moderadas ou severas neonatais como fatores de alto risco para o desenvolvimento sadio e adaptado da criança (Lewis, Duglokinski, Caputo \& Griffin, 1988; Miller, 1996; Werner, 1986). Decorrente desses problemas podem surgir outros fatores biológicos adversos, tais como hospitalização repetida ou prolongada, estando esta relacionada às doenças crônicas. Esse conjunto de fatores constitui-se, por sua vez, em fator de risco para futuras deficiências cognitivas e desordens emocionais e comportamentais da criança.

Diversos fatores de risco presentes na vida de uma mesma criança tendem a se multiplicar, antes que apenas se somar. Liaw e Brooks-Gunn (1993) sugerem que resultados de desenvolvimento insatisfatório podem ser melhor prognosticados através da combinação de diversos fatores de risco e não somente por meio de variáveis isoladas. Fatores biológicos e psicossociais podem atuar frequientemente em conjunto, tanto como causa direta ou indireta da prematuridade (O'Brien, Soliday \& McCluskeyFawcett, 1995) quanto como condições agravantes ou atenuantes do efeito da prematuridade no desenvolvimento da criança.

Na última década, foram realizados inúmeros avanços na área de Neonatologia, aumentando-se a taxa de sobrevivência de bebês nascidos prematuros com peso abaixo de 1.500 gramas. Paralelamente, diversos estudos foram realizados sobre a qualidade de vida dessas crianças vulneráveis biologicamente, a fim de verificar o impacto do fator de risco da prematuridade e de outros fatores associados na trajetória de desenvolvimento da criança. Apesar desses esforços, McCormick e Richarson (2002) salientam que continuam sendo necessários estudos sobre seguimento no curso do ciclo vital de bebês prematuros, sobreviventes e egressos de unidades de terapia intensiva neonatal.

Crianças em condições de nascimento prematuro e com baixo peso, quando comparadas a crianças com peso igual ou superior a $2.500 \mathrm{~g}$ a termo, são mais propensas a apresentar: deficiências cognitivas (Bradley \& cols., 1993; Damman \& cols., 1996; Waber \& McCornick, 1995) problemas de desempenho escolar (Taylor, Hack, Klein \& Schatschneider,
1995) e dificuldades comportamentais (Brandt, Magyary, Harmond \& Barnard, 1992; Carvalho, Linhares \& Martinez, 2001). Verifica-se ainda que quanto mais baixo o peso de nascimento, mais dificuldades ocorrem no curso do desenvolvimento (Pieuch, Leonard, Cooper \& Sehring, 1997) .

Ressalta-se, porém, que as condições neurológicas (Brazelton, 1994; Fitzhardinze \& Steven, 1992; Ross, Lipper \& Auld, 1996) e as condições ambientais familiares (Bradley \& cols., 1993; Bradley \& cols., 1994; Liaw \& Brooks-Gunn, 1993) da criança atuam de modo significativo a atenuar ou agravar o desencadeamento dessas dificuldades. Dammann e cols. (1996) verificaram que o desenvolvimento viso- motor sofre maior influência das condiçc̃es neurológicas do que das sócioeconômicas. Sansavini, Rizzardi, Alessandroni e Giovanelli (1996), por sua vez, encontraram que o baixo peso e a prematuridade são fatores relacionados com atrasos no desenvolvimento motor, cognitivo e social no primeiro ano de vida e atrasos de linguagem durante os dois primeiros anos de idade.

Se de um lado as dificuldades de desenvolvimento apresentadas por crianças pré-termo e muito baixo peso são evidentes, por outro lado existem estudos que demonstram possibilidades bem sucedidas de enfrentamento das condições adversas pela criança prematura. A relação entre os riscos biológicos enfrentados por essas crianças e o ambiente social é muito significativa, uma vez que este pode influenciar no impacto dos efeitos da prematuridade e do baixo peso ao nascer (Bradley \& cols., 1993). A condição de pobreza pode agravar o quadro de risco apresentado por essas crianças, porém, a presença de outros fatores ambientais protetores combinados aumentam a probabilidade de desencadear o processo de resiliência. Este consiste no enfrentamento de adversidades advindas de estresse interno e/ou externo ao organismo com poder de neutralizar e superar os efeitos adversos provocados por condições de risco, alcançando sucesso nas tarefas evolutivas da trajetória do.desenvolvimento (Linhares, Carvalho, Bordin \& Jorge, 1999; Yunes, Szymanski, Pereira, RalhaSimões \& Castro, 2000).

$O$ desenvolvimento futuro da criança encontra-se, portanto, associado à saúde neonatal do bebê, assim como a características maternas, variáveis do 
ambiente familiar e programas de intervenção precoce para detecção, atenuação e se possível reversão de problemas. Liaw e Brooks-Gunn (1993) salientaram a importância da intervenção precoce, com o objetivo de atenuar a tendência negativa que as crianças nascidas pré-termo e com baixo peso possuem no desenvolvimento intelectual ao longo dos três primeiros anos de vida. Os autores verificaram que o grupo de bebês pré-termo que recebeu intervenção precoce, quando comparado ao grupo controle, apresentou menos problemas de comportamento.

A intervenção pode, tanto alterar o modo como a mãe percebe o comportamento de seu filho, quanto tornar a mãe melhor observadora do bebê devido a sua participação no programa de intervenção. As mães mostram-se responsivas nos primeiros cuidados com o filho pré-termo e muito baixo peso, assim como mais sensiveis em relação aos sinais emitidos pelo bebê. Esses comportamentos maternos podem constituir-se em estratégia de compensação como forma de neutralizar os riscos aos quais os bebês estão expostos (Barrat, Roach \& Leavitt, 1996).

Nesse sentido, Novello, Degraw e Kleinnnan (1992) salientaram a importância de acompanhar o desenvolvimento de crianças com problemas neonatais, a fim de evitar que se potencializem os fatores de risco, transformando-se em problemas. Destacam-se, os trềs primeiros anos de vida de uma criança, como fase relevante para o seu desenvolvimento. As experiências vividas nesse período são decisivas tanto para o desenvolvimento cerebral, quanto para as capacidades que ela apresentará em etapas evolutivas posteriores (Shore, 1997).

A criança vulnerável nascida prematuramente pode ser bem sucedida em superar as adversidades, como demonstram M. Bradley e cols. (1994) Justifica-se, neste sentido, a importância de estudos de seguimento longitudinal que acompanhem seu processo de desenvolvimento, a fim de favorecer processos de resiliência e identificar as crianças elegíveis para intervenções especializadas, seja no âmbito educacional ou terapêutico. Linhares e cols. (2000) verificaram a importância do seguimento longitudinal do desenvolvimento de bebês nascidos pré-termo e com muito baixo peso, a fim de detectar tanto os indicadores de problemas, para revertê-los ou minimizá-los, quanto os recursos da criança e da família, para ativá- los ou potencializá-los.

O presente estudo tem o objetivo de avaliar, no curso do primeiro ano de vida, o desenvolvimento de bebês nascidos pré- termo e com muito baixo peso, participantes de um programa de seguimento longitudinal do desenvolvimento psicológico iniciado desde o período da internação do bebê na Unidade de Tratamento Intensivo Neonatal (UTIN). Esta avaliação, realizada durante seguimento longitudinal de intervenção precoce, será contextualizada pela identificação das representações e expectativas maternas, quanto ao desenvolvimento do bebê nascido prematuramente e variáveis relacionadas à experiências no ambiente familiar.

O seguimento longitudinal do desenvolvimento psicológico é oferecido regularmente a todas as crianças egressas da UTI Neonatal do HCFMRP. Segue um esquema mensal no primeiro ano de vida e, posteriormente, ocorre em intervalos trimestrais no segundo ano de vida, semestral a partir do terceiro ano e anual dos 4 aos 7 anos de idade. O objetivo do seguimento consiste em avaliar o desenvolvimento da criança e indicadores de seu contexto ambiental familiar, a fim de detectar eventuais problemas, identificar fatores de proteção, orientar quanto à promoção do desenvolvimento e encaminhar para atendimentos de intervenção especializada quando necessário.

\section{Método}

O presente estudo, com delineamento longitudinal, avaliou uma amostra de 42 bebês ( $53 \%$ meninas) com idade cronológica de 12 meses incompletos nascidos pré- termo (média $=30$ semanas de idade gestacional $[ \pm 2,58]$ ) e muito baixo peso (média= $1.073 \mathrm{~g}[ \pm 254 \mathrm{~g}]$ ), e suas respectivas mães. Os participantes integram o Programa de Seguimento Longitudinal do Desenvolvimento de Bebês Nascidos Prematuros (PSL). Este programa inicia-se, após o nascimento do bebê, durante a internação na UTIN do Setor de Neonatologia do HCFMRP-USP e se estende apos a alta hospitalar até a fase escolar.

No HCFMRP-USP ocorrem em média 1.700 nascimentos por ano, sendo que $10 \%$ destes bebês nascem pré-termo e com peso igual ou abaixo de 1500g. Destes 170 bebês, excluídos os óbitos, em média 50\% aderem ao Programa de Seguimento Lon- 
gitudinal (PSL). A amostra do presente estudo foi extraída de um total de 89 crianças integrantes do PSL, no ano de 2001, que seguiram regularmente o esquema de avaliação continuada mensal no primeiro ano de vida.

Os dados apresentados neste estudo referemse às duas avaliações do desenvolvimento dos bebês realizadas aos 6 e 11 meses de idade pós-gestacional, respectivamente, com correção da idade gestacional, utilizando-se a escala brasileira de Desenvolvimento do Comportamento da Criança-o primeiro ano de vida (EDCC) (Pinto, Vilanova \& Vieira, 1997). O ECC tem o objetivo de avaliar o desenvolvimento do comportamento da criança de 1 a 12 meses incompletos, incluindo a combinação de três eixos relacionados a: anatômico corporal (axial ou apendicular), estimulação (espontâneo ou estimulado) e função (não comunicativo ou comunicativo).

Os Quadros I e II apresentam os itens da EDCC para as referidas idades.

Quadro I: Áreas do desenvolvimento e comportamentos esperados na faixa de 6 meses de idade, de acordo com a Escala de Desenvolvimento e Comportamento da Criança (EDCC)

\begin{tabular}{|c|c|c|}
\hline \multicolumn{2}{|c|}{$\begin{array}{l}\text { ÁREAS DO DESENVOLVIMENTO } \\
\text { (EDCC) }\end{array}$} & $\begin{array}{l}\text { COMPORTAMENTOS ESPERADOS NA } \\
\text { FAIXA DE } 6 \text { MESES DE IDADE (EDCC) }\end{array}$ \\
\hline Axial Espontâneo Não Comunicativo & $\begin{array}{l}\text { E } \\
A \\
N \\
A \\
A \\
A\end{array}$ & $\begin{array}{l}\text { Fica em pé quando segurado pela cintura } \\
\text { Rola } \\
\text { Puxa para sentar-se } \\
\text { Mantém-se sentado com o apoio das mãos } \\
\text { Arrasta-se } \\
\text { Senta-se sem o apoio das mãos }\end{array}$ \\
\hline Axial Espontâneo Comunicativo & $\begin{array}{l}\text { A } \\
\text { A } \\
\text { A } \\
\text { A } \\
\text { A }\end{array}$ & $\begin{array}{l}\text { Emite sons vocálicos } \\
\text { Repete os próprios sons } \\
\text { Tem reação de esquiva frente a estranhos } \\
\text { Repete a mesma sílaba } \\
\text { Combina duas sílabas diferentes em jogo silábico }\end{array}$ \\
\hline Axial Estimulado Não Comunicativo & \begin{tabular}{|l|l|}
$\mathbf{N}$ \\
$\mathrm{N}$
\end{tabular} & $\begin{array}{l}\text { Sorri e vocaliza diante do espelho } \\
\text { Tira pano do rosto }\end{array}$ \\
\hline Axial Estimulado Comunicativo & $\begin{array}{l}\mathrm{A} \\
\mathrm{A} \\
\mathrm{A} \\
\mathrm{A}\end{array}$ & $\begin{array}{l}\text { Vira-se quando chamado pelo nome } \\
\text { Brinca de "esconde-achou" } \\
\text { Reage aos jogos corporais } \\
\text { Repete os sons feitos por outra pessoa } \\
\end{array}$ \\
\hline $\begin{array}{l}\text { Apendicular Espontâneo } \\
\text { Não Comunicativo }\end{array}$ & $\begin{array}{l}\text { E } \\
\text { N } \\
\text { A } \\
\text { A } \\
\text { A } \\
\text { A } \\
\text { A }\end{array}$ & $\begin{array}{l}\text { Percebe e explora objeto com a boca } \\
\text { Em posição prono alcança objeto } \\
\text { Apanha objeto após deixa-lo cair } \\
\text { Transfere objetos de uma mão para outra } \\
\text { Encontra objeto escondido } \\
\text { Encontra objeto intermediário } \\
\text { Retém dois pinos em uma das mãos }\end{array}$ \\
\hline Apendicular Espontâneo Comunicativo & A & Bate nos óculos, nariz e cabelos dos adultos \\
\hline $\begin{array}{l}\text { Apendicular Estimulado } \\
\text { Não Comunicativo }\end{array}$ & $\begin{array}{l}\mathrm{N} \\
\mathrm{A} \\
\mathrm{A}\end{array}$ & $\begin{array}{l}\text { Balança brinquedo sonoro } \\
\text { Chocalha brinquedo } \\
\text { Tira pinos grandes } \\
\end{array}$ \\
\hline Apendicular Estimulado Comunicativo & $\begin{array}{l}\text { A } \\
\text { A } \\
\text { A } \\
\text { A }\end{array}$ & $\begin{array}{l}\text { Para a atividade quando lhe dizem "não" } \\
\text { Responde a "vem" estendendo os braços } \\
\text { Atende a solicitação "dá" mas não solta o brinquedo } \\
\text { Atende a solicitação "dá" entregando o brinquedo }\end{array}$ \\
\hline
\end{tabular}

$\mathrm{A}=$ Aparecimento; $\mathrm{N}=$ Normalização; $\mathrm{E}=$ Estabilização 
Quadro II: Áreas do desenvolvimento e comportamentos esperados na faixa de 11 meses de idade, de acordo com a Escala de Desenvolvimento e Comportamento da Criança (EDCC)

\begin{tabular}{|c|c|c|}
\hline $\begin{array}{l}\text { ÁREAS DO DESENVOLVIMENTO } \\
\text { (EDCC) }\end{array}$ & & $\begin{array}{l}\text { COMPORTAMENTOS ESPERADOS NA } \\
\text { FAIXA DE } 11 \text { MESES DE IDADE (EDCC) }\end{array}$ \\
\hline Axial Espontâneo Não Comunicativo & $\begin{array}{l}\mathrm{E} \\
\mathrm{N} \\
\mathrm{A} \\
\mathrm{A}\end{array}$ & $\begin{array}{l}\text { Mantém em pé com o mínimo de apoio } \\
\text { Caminha com auxílio } \\
\text { Dá alguns passos sem apoio } \\
\text { Caminha independentemente }\end{array}$ \\
\hline Axial Espontâneo Comunicativo & $\begin{array}{l}\mathrm{E} \\
\mathrm{A} \\
\mathrm{A}\end{array}$ & $\begin{array}{l}\text { Repete a mesma sílaba } \\
\text { Combina duas sílabas diferentes em jogo silábico } \\
\text { Usa intencionalmente palavra com significado }\end{array}$ \\
\hline Axial Estimulado não Comunicativo & & $\begin{array}{l}\text { Não há comportamento previsto para aplicação no } \\
\text { EDCC para esta faixa etária }\end{array}$ \\
\hline Axial Estimulado Comunicativo & $\begin{array}{l}\mathrm{E} \\
\mathrm{N} \\
\mathrm{A}\end{array}$ & $\begin{array}{l}\text { Reage aos jogos corporais } \\
\text { Repete os sons feitos por outra pessoa } \\
\text { Repete as caretas feitas por outra pessoa }\end{array}$ \\
\hline $\begin{array}{l}\text { Apendicular Espontâneo } \\
\text { Não Comunicativo }\end{array}$ & $\begin{array}{l}\mathrm{N} \\
\mathrm{A}\end{array}$ & $\begin{array}{l}\text { Transfere objeto de uma mão para outra } \\
\text { Retém dois pinos em uma das mãos }\end{array}$ \\
\hline Apendicular Espontâneo Comunicativo & & $\begin{array}{l}\text { Não há comportamento previsto para aplicação no } \\
\text { EDCC para esta faixa etária }\end{array}$ \\
\hline $\begin{array}{l}\text { Apendicular Estimulado } \\
\text { Não Comunicativo }\end{array}$ & $\begin{array}{l}\mathrm{E} \\
\mathrm{N} \\
\mathrm{A} \\
\mathrm{A}\end{array}$ & $\begin{array}{l}\text { Tira pinos pequenos } \\
\text { Coloca objetos em recipiente } \\
\text { Rabisca } \\
\text { Coloca pinos grandes }\end{array}$ \\
\hline Apendicular Espontâneo Comunicativo & $\begin{array}{l}\text { N } \\
\text { A } \\
\text { A } \\
\text { A } \\
\text { A } \\
\text { A } \\
\text { A }\end{array}$ & $\begin{array}{l}\text { Atende à solicitação "dá", mas não solta o brinquedo } \\
\text { Atende à solicitação "dá", entregando o brinquedo } \\
\text { Bate palmas } \\
\text { Dá "tchau" } \\
\text { Executa gestos simples } \\
\text { Faz carinhos } \\
\text { Participa de jogos simples }\end{array}$ \\
\hline
\end{tabular}

$A=$ Aparecimento; $N=$ Normalização; $E=$ Estabilização

Considerando-se que em algumas avaliações, devido a motivo de falta à consulta ou feriados, não ocorreram exatamente aos 6 e 11 meses da criança, adotou-se o critério de incluir as avaliações realizadas mais próximas a essas idades, respeitando o limite de um mês para mais ou para menos na consulta. Todas as avaliações dos bebês eram observadas pelas mães e foram precedidas de entrevistas com as mães sobre a evolução dos bebês. Ao final da consulta, as mães recebiam uma devolutiva com informações sobre a evolução e o estado desenvolvimental atual do bebê e com orientações sobre promoção do desenvolvimento.
As mães foram entrevistadas, através de roteiro estruturado (Linhares, Oliveira \& Martinez, 1998), e os tópicos sobre antecedentes do bebê e representações sobre prematuridade e expectativas maternas em relação ao bebê foram analisados quanto ao conteúdo temático das respostas. A entrevista incluía uma Lista de Adversidades Ambientais, a fim de identificar eventos adversos no ambiente familiar tais como: instabilidade dos pais em empregos; mudanças residenciais; separação do casal; conflitos conjugais com a presença de brigas constantes e/ou com a presença de agressividade verbal e corporal 


\section{Maria Beatriz Martins Linhares}

entre os pais; doenças orgânicas crônicas na família; distúrbios psiquiátricos; distúrbios neurológicos; deficiência mental; vícios de drogas ou álcool; hospitalização de um dos pais; suicídio ou morte e delinqüência. Nos casos de as famílias nucleares contarem apenas com um dos pais, foram analisados também os seguintes itens: novo casamento ou namoro dos pais; mãe solteira durante a gravidez; ausência de contato dos pais com a criança.

As entrevistas foram transcritas e os dados organizados para posterior análise do conteúdo temático das respostas em categorias e análise quantitativa. Neste estudo, foram analisadas as questões referentes aos seguintes tópicos: concepção, gestação, condições peri- e pós-natal, representação materna acerca da prematuridade do filho, suas expectativas e sentimentos quanto a evolução e desenvolvimento do bebê e as condições psicossociais adversas no ambiente familiar. Os dados de entrevista que serão apresentados referem-se à entrevista realizada na primeira consulta após a alta hospitalar do bebê. Realizou-se também, consultas ao prontuário médico da criança junto ao hospital, para se obter dados complementares sobre a idade gestacional da criança, peso de nascimento e tipo de parto.

A análise dos dados foi processada através do pacote estatístico Statistical Package for Social Science (SPSS 10.1). Os dados foram analisados descritivamente, em termos de freqüência, porcentagem e incidência, de acordo com a natureza da variável. Procedeu-se à análise de comparação entre grupos, diferenciados, respectivamente, quanto ao gênero (meninas, $n=23$ e meninos, $n=19$ ) e peso de nascimento $(\leq 1.000 \mathrm{~g}, \mathrm{n}=16 \mathrm{e}$ de 1.001 a $1.500 \mathrm{~g}, \mathrm{n}=26)$. Os escores totais e áreas do EDCC obtidos nos grupos foram comparados através do teste $t$ de Student para amostras independentes. Procedeu-se à análise de comparação intra-grupo, levando-se em conta os escores totais de 6 e 11 meses, através do teste t para amostras pareadas. Foi realizada a análise de correlação de Pearson entre variáveis neonatais do bebê. $\mathrm{O}$ valor de $\mathrm{p} \leq 0,05$ foi adotado no estudo como significativo no tratamento estatístico dos dados.

\section{Resultados}

Pode-se observar na Tabela 1 que, na amostra, houve predomínio de nascimentos através de parto do tipo cesariana (64\%) em relação ao parto normal. Quanto à idade gestacional, cerca da metade dos bebês tinha entre 24 e 29 semanas e o restante entre 30 e 34 semanas, no máximo. Em relação ao peso de nascimento, a maior parte dos bebês $(62 \%)$ apresentava peso de nascimento entre 1.001 e 1500 gramas; o restante era constituído por bebês extremamente baixo peso (abaixo de $1.000 \mathrm{~g}$ ), predominando entre

Tabela 1: Condições perinatais dos bebês pré-termo e com muito baixo peso: frequiência (f) e porcentagem $(\%)(n=42)$

\begin{tabular}{ccc}
\hline $\begin{array}{c}\text { Condições de Gestação e } \\
\text { Nascimento }\end{array}$ & f & $\%$ \\
\hline Tipo de Parto & 15 & $36 \%$ \\
Normal & 27 & $64 \%$ \\
Cesárea & & \\
Idade Gestacional (semanas) & 18 & $43 \%$ \\
24 a 29 & 24 & $57 \%$ \\
30 a 34 & & \\
Peso de Nascimento (gramas) & 5 & $12 \%$ \\
610 a $725 \mathrm{~g}$ & 11 & $26 \%$ \\
726 a $1000 \mathrm{~g}$ & 26 & $62 \%$ \\
1.001 a $1.500 \mathrm{~g}$ &
\end{tabular}


estes a faixa de 1.000 até 725 gramas. A amostra incluía $12 \%$ de bebês micro prematuros, ou seja, igual ou abaixo de 725 gramas. Verificou-se correlação positiva significativa entre peso de nascimento e idade gestacional $(r=0,40 ; p \leq 0,01)$.

Tabela 2: Variáveis do ambiente familiar dos bebês nascidos pré-termo e com muito baixo peso: nível de escolaridade materno, renda familiar per capita e número de eventos psicossociais adversos.

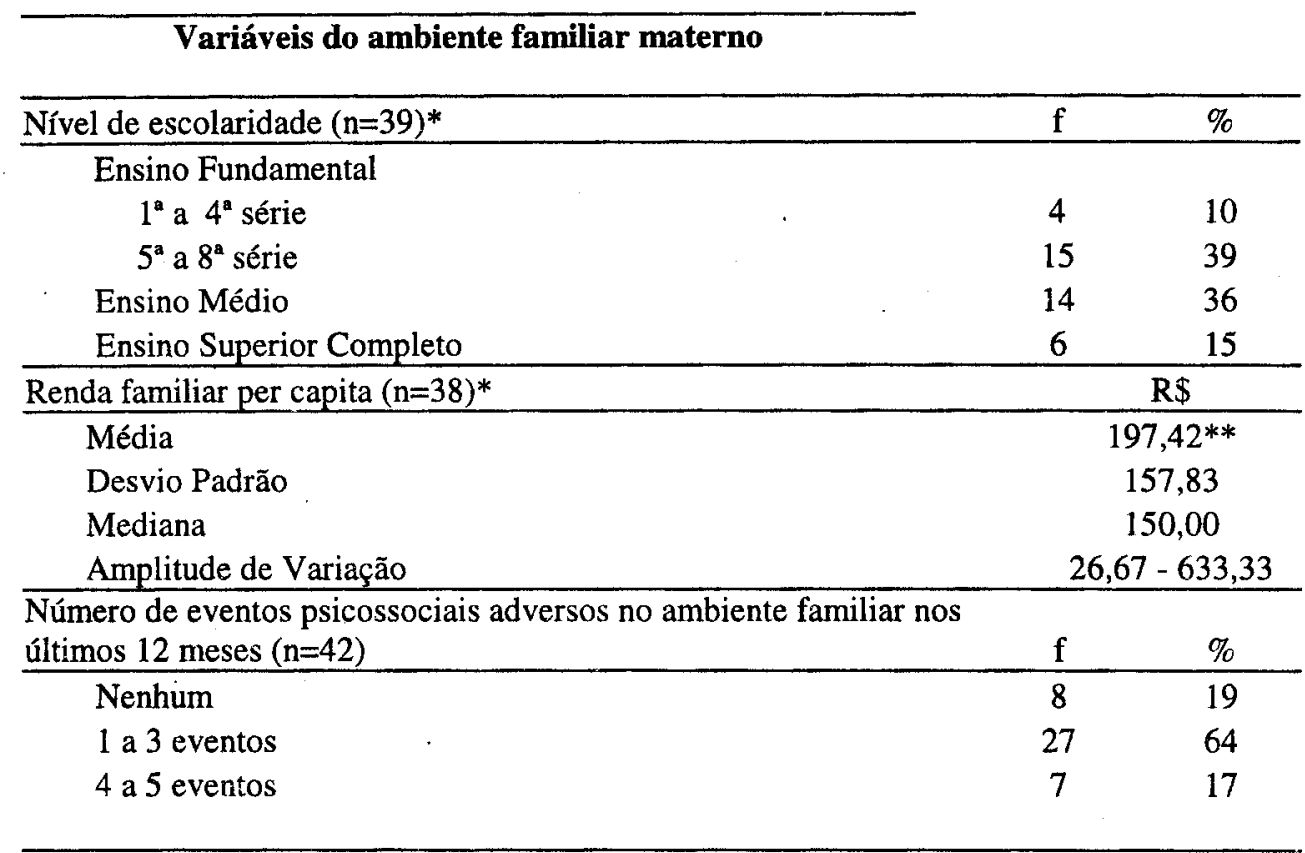

* A diferença do $n$ para o total de 42 se deve a dados prejudicado na coleta de dados.

** Salário mínimo vigente na ocasião da coleta de dados $\mathrm{R} \$$ 150,00 .

$\mathrm{Na}$ análise da Tabela 2 verifica-se que, cerca de metade das mães (49\%) possuíam nível escolar de ensino fundamental. O restante possuía, predominantemente, nível escolar de ensino médio e apenas $15 \%$ possuía nível superior. A renda mensal média per capita das famílias era de $R \$ 197,42$ ( $\pm R \$ 157,83$ ), cujo salário mínimo vigente na época da coleta de dados era igual a $R \$ 150,00$. A maior parte das famílias $(81 \%)$ apresentou uma ou mais condições psicossociais adversas, predominando de um a três eventos adversos em $64 \%$ das famílias.
Complementando esses dados, verificou-se que nas famílias nucleares com ambos os pais presentes no lar, os tipos de adversidades mais frequientemente relatados foram: mudanças residenciais, doenças crônicas, distúrbios psiquiátricos e neurológicos na família e a presença de deficiência mental e atrasos no desenvolvimento. Por outro lado, nas famílias nucleares, em que apenas um dos pais estava presente no lar, o tipo de adversidade mais frequiente foi a falta de contato do pai com a criança e a mãe. 


\section{Maria Beatriz Martins Linhares}

Tabela 3: Categorias de representação materna acerca da prematuridade do bebê: freqüência (f) e incidência (i) $(n=42)$

\begin{tabular}{lcc}
\hline Categorias de Representação Materna acerca da Prematuridade do Bebề & f & i* $^{*}$ \\
\hline Avaliação da Evolução do bebê quanto a desenvolvimento, crescimento e & & \\
comportamento & 18 & $43 \%$ \\
$\quad$ Positiva & 4 & $10 \%$ \\
$\quad$ Negativa & & \\
Expectativa do desenvolvimento do bebê & 37 & $88 \%$ \\
$\quad$ Positiva & 3 & $7 \%$ \\
$\quad$ Negativa & 2 & $5 \%$ \\
$\quad$ Duvidosa & 8 & $19 \%$ \\
Comparação com pares de bebês a termo & 5 & $12 \%$ \\
Medos quanto a sobrevivência/ hospitalização/ seqüelas/ saúde & 1 & $2 \%$ \\
Alívio devido a evolução positiva do bebê & 1 & $2 \%$ \\
Aceitação incondicional do estado de saúde do bebê & 2 & $5 \%$ \\
Reconhecimento da influência do ambiente no desenvolvimento do bebê & & \\
* i= taxa de incidência calculada pela divisão de cada categoria respectivamente pelo $\boldsymbol{n}$ de participantes \\
correspondente a 42.
\end{tabular}

A Tabela 3 revela que as mães mostravam-se com projeções otimistas em relação ao desenvolvimento do bebê, no momento da primeira entrevista do Programa de Seguimento Longitudinal do Desenvolvimento, realizada após a alta hospitalar do bebê. Verificaram-se altas incidências de respostas das mães contendo expectativa positiva acerca do desenvolvimento dos bebês ( $88 \%$ ) e predomínio de avaliação positiva sobre sua evolução após a alta hospitalar (43\%). Paralelamente, as mães apresentaram, durante a entrevista, baixa incidência de verbalizações relacionadas com medos acerca da sobrevivência, hospitalização, seqüelas ou saúde do(a) filho(a). Uma parcela de $19 \%$ das mães expressaram comparações entre o desenvolvimento de seus bebês e o de outros bebês nascidos a termo.

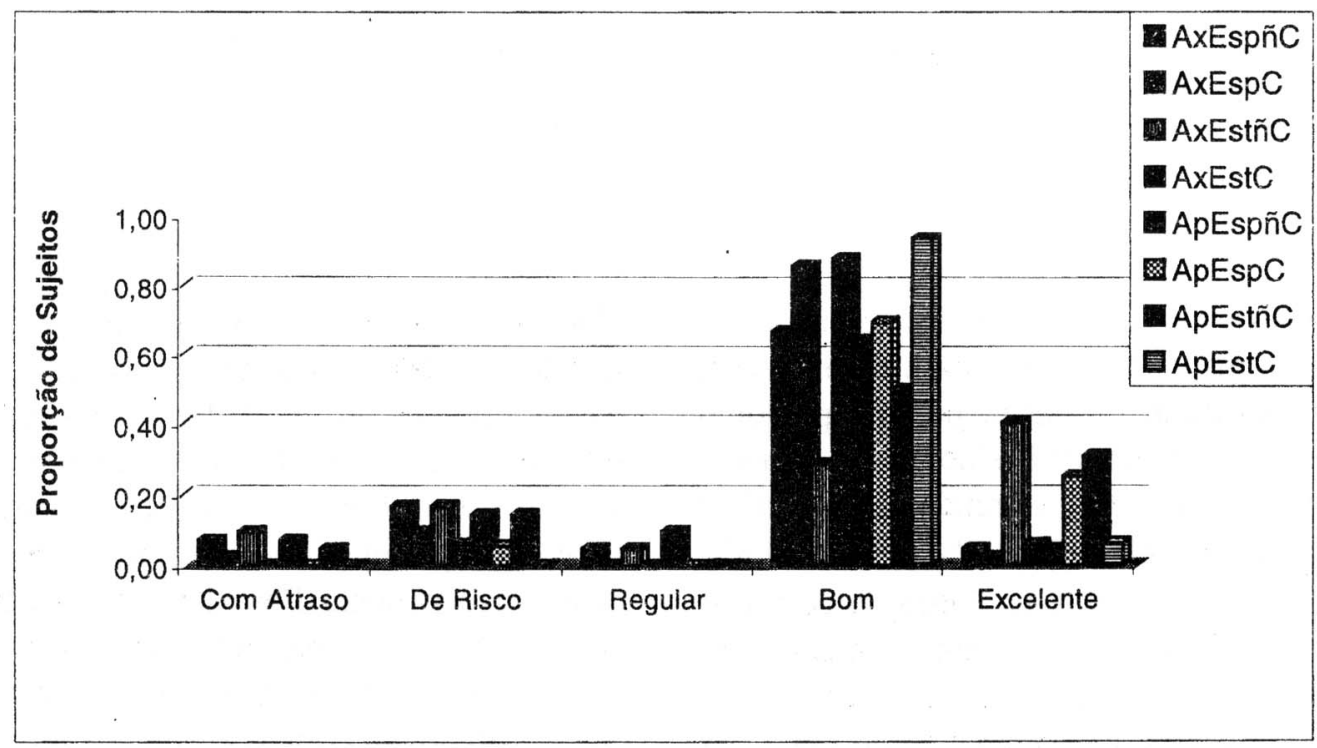

Figura 1: Escala de Desenvolvimento do Comportamento da Criança: porcentagem de sujeitos, na fase de 6 meses de idade, distribuídos por área nas diferentes classificações da escala $(n=42)$ 
$\mathrm{Na}$ Figura 1, verifica-se que na fase de 6 meses de idade, com correção de idade gestacional, a maior parte dos bebês apresentou na EDCC desempenho BOM ou EXCELENTE em torno de $70 \%$ ou mais, em todas as áreas avaliadas. Pode-se observar indícios de RISCO ou ATRASO na ordem de $30 \%$ ou menos das crianças nas seguintes áreas axial estimulado não comunicativo (27\%) axial espontâneo não comunicativo (24\%), apendicular espontâneo não comunicativo (21\%) e apendicular estimulado não comunicativo (19\%). O conjunto de comportamentos envolvido nessas áreas referem-se respectivamente a: sorrir, vocalizar diante do espelho e tirar o pano do rosto; ficar em pé quando segurado pela cintura, rolar e puxar para sentar; perceber e explorar objeto com a boca, em posição prona alcançar objeto e apanhar objeto após deixa-lo cair; balançar brinquedo sonoro, chocalhar brinquedo e tira pinos grandes.

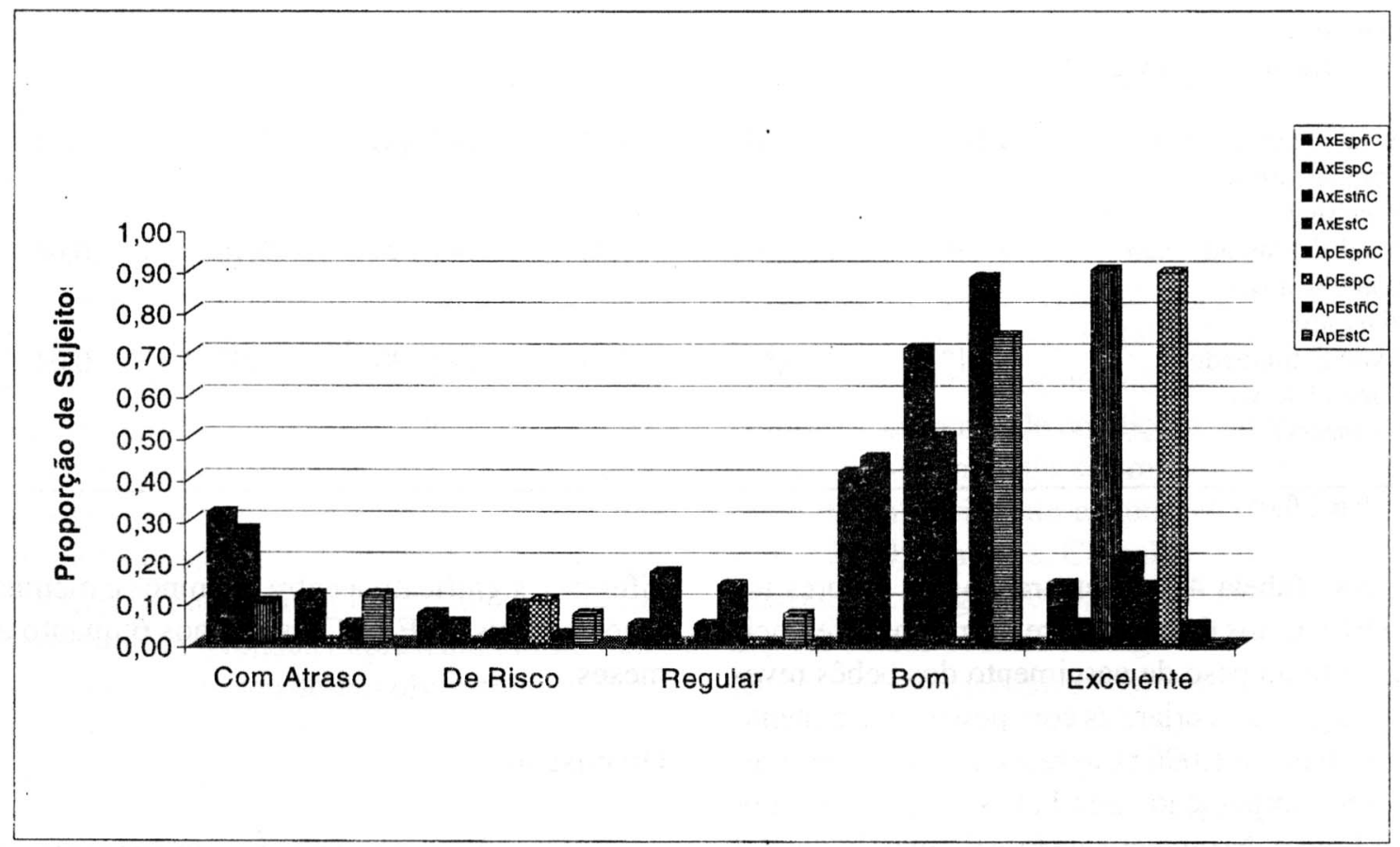

Figura 2: Escala de Desenvolvimento do Comportamento da Criança: porcentagem de sujeitos, na fase de 11 meses de idade, distribuídos por área nas diferentes classificações da escala $(n=42)$

Os dados apresentados na Figura 2 demonstram a mesma tendência observada na fase de 6 meses de idade corrigida. Aos 11 meses, a maior parte dos bebês foi classificada na EDCC predominantemente como BOM e EXCELENTE (igual ou acima de 64\%), com exceção das áreas axial espontâneo não comunicativo e axial espontâneo comunicativo. Embora a maior parte das crianças tenha apresentado resultados favoráveis, nestas áreas verificaram-se indícios de ATRASO ou RISCO, em torno de $39 \%$ e $33 \%$, respectivamente. Os comportamentos envolvidos, na área axial espontâneo não comunicativo incluem manter-se em pé com apoio mínimo e caminhar com auxílio e, na área e axial espontâneo comunicativo, por sua vez, incluem repetir os pró- prios sons e repetir a mesma sílaba.

A análise da comparação intra-grupo, entre os escores totais alcançados na EDCC nos dois momentos do primeiro ano de vida, não revelou diferença significativa (aos 6 meses, $X=161[ \pm 97]$ e aos 11 meses, $X=136[ \pm 97] ; F=[41] 1,18 ; p=0,24)$; ou seja, o desempenho da EDCC alcançado na fase desenvolvimental aos 6 meses apresentou pontuação semelhante à encontrada aos 11 meses de idade. 
Tabela 4: Desempenho na escala EDCC (escore total e escores por áreas) - resultados significativos obtidos através da comparação entre dois grupos diferenciados pelo peso de nascimento (Grupo $1==1.000 \mathrm{~g}$ e Grupo $2=1.001$ a $1.500 \mathrm{~g})$.

\begin{tabular}{|c|c|c|c|c|c|c|}
\hline \multirow[t]{2}{*}{ EDCC } & \multicolumn{2}{|c|}{$\begin{array}{c}\text { Grupo 1 } \\
\leq 1.000 \mathrm{~g} \\
(\mathrm{n}=16)\end{array}$} & \multicolumn{2}{|c|}{$\begin{array}{c}\text { Grupo 2 } \\
1.001 \text { a 1.500g } \\
(n=26)\end{array}$} & \multirow[t]{2}{*}{$\mathbf{t}$} & \multirow[t]{2}{*}{$\begin{array}{c}\text { Valor de } \mathbf{p} \\
\quad \leq^{*}\end{array}$} \\
\hline & Média & SD & Média & SD & & \\
\hline \multicolumn{7}{|l|}{ Escore Total } \\
\hline $\begin{array}{l}\text { Escore Total } \\
(6 \text { meses) } \\
\quad \text { Escores por Àreas }\end{array}$ & $123^{*}$ & $( \pm 85)$ & 184 & $( \pm 99)$ & 2,05 & 0,05 \\
\hline $\begin{array}{l}\text { Axial Espontâneo Não } \\
\text { Comunicativo } \\
\text { (6 meses) }\end{array}$ & $1,50^{*}$ & $( \pm 0,52)$ & 1,92 & $( \pm 0,48)$ & 2,69 & 0,01 \\
\hline $\begin{array}{l}\text { Axial Estimulado Não } \\
\text { Comunicativo } \\
\text { (6 meses) }\end{array}$ & $1,75^{*}$ & $( \pm 0,77)$ & 2,31 & $( \pm 0,84)$ & 2,16 & 0,04 \\
\hline $\begin{array}{l}\text { Axial Estimulado } \\
\text { Comunicativo } \\
\text { (11 meses) }\end{array}$ & $1,94 *$ & $( \pm 0,57)$ & 2,27 & $( \pm 0,45)$ & 2,08 & 0,04 \\
\hline
\end{tabular}

Na Tabela 4, a comparação dos escores totais na EDCC aos 6 meses entre os grupos diferenciados quanto ao peso de nascimento dos bebês revelou que, o grupo de crianças com peso de nascimento igual ou abaixo a $1.000 \mathrm{~g}$ ), apresentaram escores menores em comparação aos bebês com peso entre $1.001 \mathrm{e} 1.500 \mathrm{~g}$. No entanto, na fase desenvolvimental de 11 meses não foi encontrada diferença significativa entre os dois referidos grupos.

Com relação aos escores específicos, comparando-se os grupos foram encontradas diferenças significativas em três áreas comportamentais da EDCC. Os bebês com menor peso ao nascer apresentaram desempenho mais rebaixado, em comparação com os bebês de maior peso, nas seguintes áreas: a) aos 6 meses, axial espontânea não comunicativa (comportamento de permanecer em pé segurado pela cintura) e axial estimulado não comunicativo (comportamentos de sorrir, vocalizar frente ao espelho e tirar pano do rosto); b) aos 11 meses, axial estimulado comunicativo (comportamentos de reagir a jogos corporais e repetir sons feitos por outras pessoas).

Com relação à comparação entre grupos diferenciados por gênero, no entanto, não foi encontrada diferença significativa entre meninos e meninas nos escores totais da EDCC, tanto aos 6 quanto aos 11 meses.

\section{Discussão}

Com base nos achados do presente estudo, verificou-se que a avaliação do desenvolvimento de 42 crianças nascidas pré-termo e com peso ao nascer igual ou abaixo de $1500 \mathrm{~g}$, que participavam do Programa de Seguimento Longitudinal do HCFMRPUSP desde sua alta hospitalar, revelou resultados positivos quanto à trajetória evolutiva das crianças durante o primeiro ano de vida.

Quanto às condições perinatais, verificou-se que os bebês nasceram predominantemente de parto do tipo cesariana. $O$ peso de nascimento dos bebês, variou entre 610 e $1500 \mathrm{~g}$, sendo uma parcela de $38 \%$ de bebês nascidos com peso igual ou abaixo de $1000 \mathrm{~g}$, o que caracteriza um grupo de crianças com alto risco para problemas de desenvolvimento. A marcada vulnerabilidade biológica foi observada neste grupo de sobreviventes, considerando-se os fatores da prematuridade e do muito baixo peso ao nascer dos bebês. 
A avaliação do desenvolvimento das crianças através da escala brasileira Desenvolvimento do Comportamento da Criança - o primeiro ano de vida indicou que, a maior parte das crianças de ambos os sexos, com a idade corrigida apresentou desempenho BOM ou EXCELENTE em todos os comportamentos analisados nas diferentes áreas da escala, nas faixas de idade de 6 e 11 meses, respectivamente. Na primeira faixa, uma parcela inferior a $20 \%$ das crianças apresentou indícios de risco ou atraso em comportamentos preparatórios para andar e sentar (manter postura e equilíbrio para ficar em pé) e comportamentos pró-sociais (sorrir, vocalizar diante o espelho e tirar o pano do rosto). Na segunda faixa, uma parcela em torno de $30 \%$, no máximo, apresentou indícios de atraso nos comportamentos preparativos, ou propriamente, do andar e comportamentos verbais. $\mathrm{O}$ índice de crianças com indicadores de risco ou atraso encontrado na amostra estudada mostra-se inferior ao encontrado por Halpern, Giuglian, Victoria, Barros e Horta (2000) em uma amostra de crianças aos 12 meses sem risco estabelecido nascidas nos hospitais de Pelotas. Pode-se afirmar, portanto, que com a correção da idade no primeiro ano de vida, as crianças pré-termo avaliadas apresentaram no conjunto boa evolução do seu desenvolvimento.

Salienta-se, no entanto, a necessidade de estimular aquisições específicas significativas enquanto marcos do desenvolvimento no primeiro ano de vida, tais como sorriso, verbalizações, motricidade ampla e jogo de esconde-descobre, este relacionado à noção de permanência do objeto. Esses comportamentos são relevantes para o desenvolvimento motor, social, da linguagem e cognitivo da criança, que será construído com os recursos pessoais da criança e as experiências advindas da interação social estabelecida com os cuidadores primários na trajetória do desenvolvimento.

Os achados do presente estudo confirmam estudos anteriores, como por exemplo de Pieuch e cols. (1997), de que quanto mais baixo o peso de nascimento maior a probabilidade de problemas no desenvolvimento. Pode-se verificar especificamente diferença significativa entre as crianças nascidas extremo baixo peso (abaixo de $1.000 \mathrm{~g}$ ) e crianças nascidas entre 1.001 e 1.500 gramas em comportamentos esperados para 6 meses de idade na EDCC. Porém, o mesmo não ocorreu aos 11 meses de idade. Verificou-se, portanto, que ao nascer, quanto mais baixo peso maior a vulnerabilidade do bebê para as aquisições desenvolvimentais relevantes dos primeiros meses de vida sugerindo a necessidade de suporte continuado para promoção do desenvolvimento. Por outro lado, observa-se que no contexto de um programa de intervenção precoce, com suporte psicológico, os bebês mais vulneráveis demonstraram sinais de recuperação de falhas desenvolvimentais ao final do primeiro ano de vida.

Com relação às condições ambientais familiares, que constitui-se em contexto de desenvolvimento dos bebês, verificou-se que, segundo informações das mães, $81 \%$ das famílias apresentaram uma ou mais condições adversas presentes, predominando de um a três eventos ambientais adversos. Os tipos de adversidades envolviam instabilidade de moradia ou presença de distúrbios ou enfermidades crônicas na família. A presença de condições adversas no ambiente familiar constituiu-se em fator de risco e ameaça ao desenvolvimento adaptativo da criança (Carvalho, 2000; Linhares, Carvalho, Bordin, Chimello, Martinez \& Jorge, 2000; Rutter, 1987). Quando se associa o fator de risco biológico da prematuridade a fatores psicossociais adversos, tem-se constituída uma situação de múltiplo risco, como salientam Liaw e Brooks-Gunn (1993). Considerando-se que, além da vulnerabilidade orgânica das crianças do presente estudo, estas tambem estavam expostas a adversidades ambientais, redobra-se a importância de ativar fatores protetores contra a adversidade em potencial, a fim de neutralizar os riscos e garantir a trajetória adaptativa desse grupo de crianças. Verifica-se que, apesar de as crianças estudadas pertencerem a um grupo de risco com vulnerabilidade biológica associada a ambientes com eventos psicossociais estressores e baixa escolaridade materna e baixa renda, elas apresentaram indícios favoráveis ao seu desenvolvimento no primeiro ano de vida. Esses achados indicam que precocemente no primeiro ano de vida observou-se que na maior parte das crianças ocorreu sinais do processo de resiliência, ou seja, de superação das adversidades e enfrentamento bem sucedido das tarefas evolutivas iniciais.

Algumas considerações sobre esses achados podem levar a três aspectos que podem ter influenci- 


\section{Maria Beatriz Martins Linhares}

ado o desempenho positivo da maior parte das crianças na EDCC, seja de forma isolada ou combinada. Primeiramente, um aspecto que pode ter contribuído para os resultados favoráveis do desenvolvimento dos bebês relaciona-se ao fato de as mães, após a alta hospitalar dos bebês, revelaram perspectivas otimistas em relação ao desenvolvimento do bebê; $85 \%$ das mães tinham expectativas positivas quanto à evolução do bebê e $38 \%$ já os avaliavam positivamente. Apesar de o contexto ambiental familiar apresentar eventos psicossociais adversos, as mães apresentaram concepções positivas sobre os bebês, ao mesmo tempo em que investiam no seu desenvolvimento através da adesão e comparecimento mensal ao programa de seguimento longitudinal no primeiro ano de vida dos bebês.

Em segundo lugar, o próprio seguimento psicológico longitudinal desses bebês pode também ter contribuído como mecanismo protetor, pois potencialmente ativava na criança e na família recursos contra as adversidades ao desenvolvimento, na medida em que realizava detecção precoce de problemas e oferecia orientações promotoras do desenvolvimento. Liaw e Brooks-Gunn, (1993) constataram em seu estudo que as crianças pré-termo e com baixo peso quando são acompanhadas ao longo do seu desenvolvimento apresentam menos problemas de comportamento do que crianças não acompanhadas. A importância de se acompanhar o desenvolvimento de crianças de risco também foi destacada por Noveilo e cols (1992). Tendo isso em vista, sugere-se que os resultados positivos, obtidos neste estudo, podem ser reflexos da inserção destas crianças e mães no referido Programa de Seguimento Longitudinal. A orientação $\mathrm{e} O$ apoio psicossocial dado às mães podem alertar sobre as possíveis dificuldades que esses bebês poderão ter quanto a percepção e reação aos estímulos ambientais e informar sobre mediação social do desenvolvimento e aprendizagem do seu filho. $O$ acompanhamento permite detectar, mediante o resultado das avaliações, possíveis problemas no desenvolvimento e dessa forma, planejar a intervenção necessária evitando-se ou atenuando-se riscos.

Em terceiro lugar, destaca-se que o seguimento psicológico a mães e bebês foi realizados por uma mesma psicóloga, de forma continuada e longitudinal desde o período da internação do bebê na UTI
Neonatal até o seu seguimento ambulatorial. Esse aspecto pode ser entendido como fonte de suporte psicossocial estável e fortalecedora do vínculo entre as díades mãe e bębê e a profissional responsável pelo atendimento das mesmas. Neste caso, constituise portanto em um contexto desenvolvimental assistido profissionalmente, positivamente diferenciado no atendimento da criança vulnerável devido a prematuridade do nascimento. Pode-se supor, portanto, que na amostra de crianças estudadas ocorreu o interjogo entre fatores de risco, mecanismos protetores e processos promotores do desenvolvimento.

Finalizando-se, uma vez estabelecido o risco da prematuridade, deve-se portanto avaliar e intervir preventivamente para ativar recursos da criança e estimular a proteção mediada adequadamente nos contextos dos ambientes familiar e educacional e, se preciso, no contexto terapêutico. Para tanto, na área da Saúde, deve-se assegurar programas estruturados e continuados de intervenção preventiva na modalidade de seguimento longitudinal multidisciplinar, de preferência iniciado desde a internação do bebê na UTI Neonatal, para promover qualidade de vida aos bebês sobreviventes nascidos prematuramente, facilitando-se o desencadeamento de processos de resiliência na sua trajetória do desenvolvimento.

\section{Referências Bibliográficas}

Barrat, M.S., Roach, M.A. \& Leavitt, L.A. (1996). The impact of low-risk prematrity on maternal behaviour and toddler outcomes. International Journal of Behavioral Development, 19(3), 581-602.

Bradley, R.H., Whiteside, L., Caldwell B.M., Casey, P.H., Kelleher, K., Pope, S., Swanson, M., Barret, K. \& Cross, D. (1993). Maternal IQ, the home enviroment, and child IQ in low birthweight, premature children. International Journal of Behavioral Development, 16, 61-74.

Bradley, R.H., Whiteside, L., Mundfrom, D., Casey, P.H., Kelleher, K.J. \& Pope, S.K. (1994). Early indications of resilience and their relation to experiences in the home enviroment of low birthweight, pemature children living in poverty. 
Child Development, 65, 346-360.

Brandt, P., Magyary, D., Harmond, M. \& Barnard, K. (1992). Learning and behavioral-emotional problems of children born preterm at second grade. Journal of Pediatric Psychology, 17, 291-311.

Brazelton, T.B. (1994). Momentos decisivos do desenvolvimento infantil. São Paulo: Martins Fontes.

Carvalho, A.E.V. (2000). Sobrevivência e qualidade de vida: história de desenvolvimento, aprendizagem e adaptação psicossocial de crianças nascidas pré-termo e com muito baixo peso $(<1500 \mathrm{~g})$ em comparação a crianças nascidas a termo. Dissertação de Mestrado em Saúde Mental, Faculdade de Medicina de Ribeirão Preto-USP.

Carvalho, A.E.V., Linhares, M.B.M. \& Martinez, F.E. (2001). História de desenvolvimento e comportamento de crianças prematuras de baixo peso $(<1500 \mathrm{~g})$. Psicologia: Reflexão e Crítica, 14 (1), 1-33.

Damman, O., Waither, H., Allers, B., Schröder, M., Drescher, J., Lutz, D., Veelken, N. \& Schulte, F.J. (1996). Development of a regional cohort of very low-birthweight children at six years cognitive abilities are associated with neurological disability and social background. Developmental Medicine and Child Neurology, 38, 97-108.

Fitzhardinze, P.M. \& Steven, E.M. (1992). The smallfor-date infant II. Neurological and intelectual. sequelas. Pediatrics, 50 (1), 50-57.

Halpern, R., Giuglian, E.R.J., Victoria-Barros, F.C. \& Horta, B.L. (2000). Fatores de risco para suspeitas de atraso no desenvolvimento neuropsicomotor aos 12 meses de vida. Jornal de Pediatria, 76 (6), 421-428.

Lewis, R.J., Duglokinski, Caputo L.M. \& Griffin R.B. (1988). Children at risk for emotional disorders: risk and dimensions. Clinical Psychology Review, 8, 417-440.

Liaw, F.R. \& Brooks-Gunn, J. (1993). Patterns of low-birth-weight children's cognitive develop- ment. Developmental Psychology, 29 (6), 10241035.

Linhares, M.B.M., Carvalho, A.E.V., Bordin, M.B.M. \& Jorge, S.M. (1999). Suporte psicológico ao desenvolvimento de bebês prematuros com peso de nascimento de <1500g: na UTI Neonatal e no seguimento longitudinal. Temas em Psicologia da $S B P, 8(3), 245-262$.

Linhares, M.B.M., Carvalho, A.E.V., Bordin, M.B.M., Chimello, J.T., Martinez, F.E. \& Jorge, S.M. (2000). Prematuridade e muito baixo peso ao nascer como fator de risco ao desenvolvimento psicológico da criança. Cadernos de Psicologia e Educação - Paidéia, 10(12), 60-69.

Linhares, M.B.M., Oliveira, M.B.O. \& Martinez, F.E. (1998). Seguimento psicológico de RN baixo peso nos seis primeiros anos de vida. Resumos do II Congresso Brasileiro de Psicologia do Desenvolvimento, 34 .

McCormick, M.C. \& Richarson, D.K. (2002). Premature infants grow up. The New England Journal of Medicine, 346(17), 197-198.

Miller, M. (1996). Relevance of resilience to individuals with learning disabilities. International Journal of Disability and Education, 43(3), 255269.

Novello, A.C., Degraw, C.E. \& Kleinnnan, D.V. (1992). Health children ready to learn: an essencial collaboration beteween health and education. Public Health Reports, 107(1), 3-10.

O'Brien, M., Soliday, E. \& McCluskey-Fawcett, K. (1995). Prematurity and the Neonatal Intensive Care Unit. Em: M. C. Roberts. Handbook of Pediatric Psychology (pp. 463-478). New York: The Guilford Press.

Pieuch, R. E., Leonard, C.H., Cooper, B.A. \& Shering, S.A. (1997). Outcome of extremely low birth weight (500 to 900 grams) over 12-year period. Pediatrics, 100, 4, 633-639.

Pinto, E.B., Vilanova, L.C.P. \& Vieira, R.M. (1997). $O$ desenvolvimento da criança no primeiro ano de vida - padronização de uma escala para 
72 Maria Beatriz Martins Linhares

avaliação e acompanhamento. São Paulo: Casa do Psicólogo.

Ross, G., Lipper, E. \& Auld, P.A.M. (1996). Cognitive abilities and early precursors of learning disabilities in very-low birthweight children with normal inteligence and normal neurological status. International Journal of Behavioral Development, 19(3), 563-580.

Rutter, M. (1987). Psychosocial resilience and protective mechanism. Armerican Journal OrthoPsychiatry, 57, 316-331.

Sansavini, A., Rizzardi, M., Alessandroni, R. \& Giovanelli, G. (1996). The development of italian low and very low birthweight infants from birth to 5 years: the role of biological and social risks. International Journal of Behavioral Development, 19 (3), 533-547.

Shore, R. (1997). Rethinking the Brain-New Insights into Early Development. New York: Families and Work Institute.

Taylor, H.G., Hack, M., Klein, N. \& Schatschneider, C. (1995). Achievement inchildren with birth weight less than 750 with normal cognitive abilities: evidence for specific learning disabilities. Journal of Pediatric Psychology, 20(6), 703719.

Waber, D.P. \& McCornick, M.C. (1995). Late neuropsychological outcomes in pretems infants of normal IQ: selective vulnerability of the visual system. Journal of Pediatric Psychology, 20(6), 721-735.

Werner, E.E. (1986). A longitudinal study of perinatal risk. Em: D.C. Farran \& J.D. McKinney (Orgs.), Risk in intellectual and psychosocial development (pp. 61-81) Orlando: Academic Press.

Yunes, M.A.M., Szymanski, H., Pereira, A.M.S., Ralha-Simões, H. \& Castro, M.A.C.D. (2000) Em J. Tavares (Org.). Resiliência e Educação. São Paulo: Cortez Editora.

Apoio financeiro: FAPESP/ CNPq/ FAEPA-

HCFMRP 